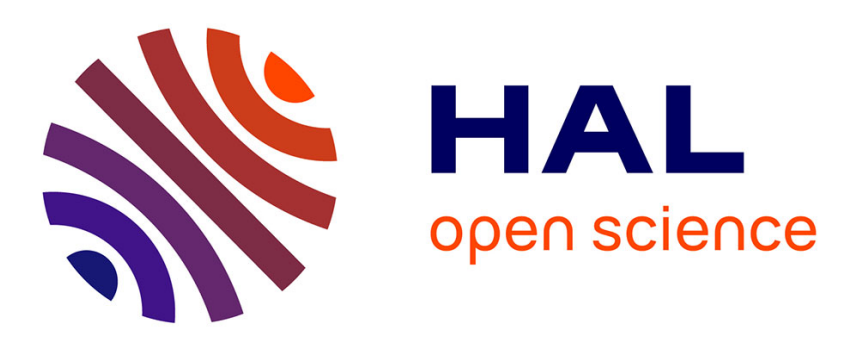

\title{
Laser frequency stabilization using folded cavity and mirror reflectivity tuning
}

Xing Liu, K Cassou, R Chiche, K Dupraz, P Favier, R Flaminio, Y Honda, A Martens, Christophe Michel, L Pinard, et al.

\section{- To cite this version:}

Xing Liu, K Cassou, R Chiche, K Dupraz, $\mathrm{P}$ Favier, et al.. Laser frequency stabilization using folded cavity and mirror reflectivity tuning. Optics Communications, 2016, 369, 10.1016/j.optcom.2016.02.028 . in2p3-01357302

HAL Id: in2p3-01357302

https://hal.in2p3.fr/in2p3-01357302

Submitted on 29 Aug 2016

HAL is a multi-disciplinary open access archive for the deposit and dissemination of scientific research documents, whether they are published or not. The documents may come from teaching and research institutions in France or abroad, or from public or private research centers.
L'archive ouverte pluridisciplinaire HAL, est destinée au dépôt et à la diffusion de documents scientifiques de niveau recherche, publiés ou non, émanant des établissements d'enseignement et de recherche français ou étrangers, des laboratoires publics ou privés. 


\title{
Laser frequency stabilization using folded cavity and mirror reflectivity tuning
}

\author{
X. Liu ${ }^{\mathrm{a}, \mathrm{b}, *}$, K. Cassou ${ }^{\mathrm{a}}$, R. Chiche ${ }^{\mathrm{a}}$, K. Dupraz ${ }^{\mathrm{a}}$, P. Favier ${ }^{\mathrm{a}}$, R. Flaminio ${ }^{\mathrm{c}}$, Y. Honda ${ }^{\mathrm{d}}$, \\ W.H. Huang ${ }^{b}$, A. Martens ${ }^{\text {a }}$, C. Michel ${ }^{\text {c }}$, L. Pinard ${ }^{\text {c }}$, B. Sassolas ${ }^{\text {, }}$, V. Soskov ${ }^{\text {a }}$ C.X. Tang , \\ F. Zomer ${ }^{\text {a }}$ \\ a LAL, CNRS-IN2P3, Université Paris-Sud, Centre Scientifique d'Orsay, Bât. 200, BP 34, 91898 Orsay Cedex, France \\ ${ }^{\mathrm{b}}$ Department of Engineering Physics, Tsinghua University, Beijing 100084, China \\ c Laboratoire des Matériaux Avancés, CNRS/IN2P3, 7 Avenue Pierre de Coubertin, 69622 Villeurbanne Cedex, France \\ ${ }^{\mathrm{d}}$ High Energy Accelerator Research Organization (KEK), Accelerator Institute, Oho 1-1, Tsukuba, Ibaraki 305-0801, Japan
}

\section{A R T I C L E I N F O}

\section{Article history:}

Received 12 October 2015

Received in revised form

10 February 2016

Accepted 15 February 2016

\section{Keywords:}

Polarization

Optical cavity

Stabilization

Lock

\begin{abstract}
A B S T R A C T
A new method of laser frequency stabilization using polarization property of an optical cavity is proposed. In a standard Fabry-Perot cavity, the coating layers thickness of cavity mirrors is calculated to obtain the same phase shift for s- and p-wave but a slight detuning from the nominal thickness can produce s- and p-wave phase detuning. As a result, each wave accumulates a different round-trip phase shift and resonates at a different frequency. Using this polarization property, an error signal is generated by a simple setup consisting of a quarter wave-plate rotated at $45^{\circ}$, a polarizing beam splitter and two photodiodes. This method exhibits similar error signal as the Pound-Drever-Hall technique but without need for any frequency modulation. Lock theory and experimental results are presented in this paper.
\end{abstract}

(c) 2016 Elsevier B.V. All rights reserved.

\section{Introduction}

Fabry-Perot cavities have a variety of applications, such as high $\mathrm{X} / \gamma$ ray flux production [1-3], high-harmonic generation [4,5], polarized positron generation [6,7], laser-wire and e-beam polarization measurement $[8,9]$.

Frequency locking is needed to maintain a cavity in resonance. An error signal that is proportional to the difference between the laser and the cavity resonance frequencies [10] is thus required. This error signal is used in a feedback system to actuate the laser repetition frequency to compensate the fluctuation between it and the cavity free spectral range. A common method for laser frequency stabilization is the Pound-Drever-Hall ( $\mathrm{PDH}$ ) method $[11,12]$ where the laser frequency is modulated to create non-resonant sidebands in the laser spectrum to produce an error signal. In a similar way, tilt locking uses non-resonant spatial modes produced by a misalignment of the laser beam with respect to the cavity as sidebands $[10,13]$. In addition, Hansch-Couillaud method introduces a polarizer inside the cavity to change the polarization of the laser beam to produce an error signal [14]. A variation of this

\footnotetext{
* Corresponding author at: LAL, CNRS-IN2P3, Université Paris-Sud, Centre Scientifique d'Orsay, Bât. 200, BP 34, 91898 Orsay Cedex, France.

E-mail address: liuxing09thu@gmail.com (X. Liu).
}

method has been realized in Ref. [15] without the addition of a polarizer, exploiting the unique property of non-planar cavities to have a different round-trip phase shift for left and right circularpolarization waves. Finally, the intrinsic birefringence of the mirrors coating of an optical cavity can also be used to produce an error signal [16].

A tiny change of the coating layer thickness of the cavity mirrors leads to s- and p-wave phase detuning. In the same way, if the incidence angle of cavity mirror is different from the nominal one for which the coating has been optimized, s- and p-wave also exhibit different phase shifts although the cavity is made of an even number of mirrors [17]. As a result, s- and p-wave accumulate a different phase after one round-trip in the cavity, i.e. they resonate at different frequencies. In this paper, a new method is proposed to implement a simple error signal by using this polarization property. To the best of our knowledge, this lock method using the mirror reflection phase shift for different polarization has never been implemented before. Contrary to the method of Ref. [16], the method presented in this paper is not usable for twomirror cavities since it uses a phase shift between the $s$ and $p$ waves reflection coefficient. However, the error signal obtained with the method of Ref. [16] depends on the azimuth orientation of the cavity mirrors [18], while it only depends on the incident angles which are fixed by the cavity geometry in the method described in this paper. The implementation of the method of Ref. 
[16] in the case of four-mirrors cavity may therefore be more complex.

This paper is organized as follows. Phase detuning of the mirror coating is presented in Section 2. The scheme of locking method is described in Section 3. The experimental results are in Section 4.

\section{Phase detuning of the mirror coating}

The phase shift difference between s- and p-wave can be controlled by adjusting the last layer of a quarter-wave layers stack like $(H L)^{N} H L_{\alpha}$, where $H$ is a high index quarter-wave layer (e.g. $\mathrm{Ta}_{2} \mathrm{O}_{5}$ ), $L$ is a low index quarter-wave layer (e.g. $\mathrm{SiO}_{2}$ ), $N$ is the number of periods, and $L_{\alpha}$ is the special tuning layer where $\alpha$ is the tuning coefficient of the last coating thickness. The matrix theory developed in Ref. [19] allows us to compute the normalized electric and magnetic fields $\mathbf{B}$ and $\mathbf{C}$ at the front surface of the multilayer and parallel to it. All the optical properties can be deduced from these two quantities.

The characteristic matrix of a non-absorbing layer with refraction index $n$ and a thickness $e$ is defined by

$R(\delta, \eta)=\left(\begin{array}{cc}\cos \delta & i \sin \delta / \eta \\ i \eta \sin \delta & \cos \delta\end{array}\right)$

where $\delta=2 \pi n e \cos \theta / \lambda, \theta=\arcsin \left(\sin \theta_{0} / n\right), \theta_{0}$ is the angle of incidence in vacuum, $\eta$ is the optical admittance given by $\eta=\eta_{0} n \cos \theta$ or $\eta=\eta_{0} n / \cos \theta$ for s- and p-wave respectively, and $\eta_{0}=\sqrt{\varepsilon_{0} / \mu_{0}}$ is the vacuum admittance. The dependence of $\eta$ with polarization comes from different boundary conditions for $\mathbf{B}$ and $\mathbf{C}$ at the interface in both polarization planes.

A multilayer stack can be represented by an equivalent surface with an admittance $\mathrm{Y}$ given by

$Y=\frac{\|\mathbf{C}\|}{\|\mathbf{B}\|}$

$\left(\begin{array}{l}B \\ C\end{array}\right)=R\left(\delta_{L}^{\prime}, \eta_{L}\right) R\left(\frac{\pi}{2}, \eta_{H}\right)\left[R\left(\frac{\pi}{2}, \eta_{L}\right) R\left(\frac{\pi}{2}, \eta_{H}\right)\right]^{N}\left(\begin{array}{c}1 \\ \eta_{\text {sub }}\end{array}\right)$.

The subscripts $H, L$ and $s u b$ stand for high index material, low index material and the substrate respectively. The first matrix is the characteristic matrix of the tuned layer, for which $\delta_{L}^{\prime}=\alpha \pi / 2$, the second matrix is for the last high index quarter-wave layer and the last one is for $N$ periods of quarter-wave layers $(H L)$.

The amplitude reflection coefficient is calculated from $r=\left(\eta_{0}-Y\right) /\left(\eta_{0}+Y\right)$. Thus, the phase shift on reflection can be deduced from

$\tan \varphi=\frac{\operatorname{Im}(r)}{\operatorname{Re}(r)}$

where

$\operatorname{Im}(r)=-2 \cos \delta_{L}^{\prime} \sin \delta_{L}^{\prime} \eta_{0}\left[\eta_{\text {sub }}^{2} \eta_{L} / \eta_{H}^{2}\left(\eta_{L} / \eta_{H}\right)^{2 N}-\eta_{H}^{2} / \eta_{L}\left(\eta_{H} / \eta_{L}\right)^{2 N}\right]$

and

$$
\begin{aligned}
\operatorname{Re}(r)= & \eta_{0}^{2}\left[\sin ^{2} \delta_{L}^{\prime} \eta_{H}^{2} / \eta_{L}^{2}\left(\eta_{H} / \eta_{L}\right)^{2 N}+\cos ^{2} \delta_{L}^{\prime} \eta_{s u b}^{2} / \eta_{H}^{2}\left(\eta_{L} / \eta_{H}\right)^{2 N}\right] \\
& -\cos ^{2} \delta_{L}^{\prime} \eta_{H}^{2}\left(\eta_{H} / \eta_{L}\right)^{2 N}-\sin ^{2} \delta_{L}^{\prime} \eta_{s u b}^{2}\left(\eta_{H} / \eta_{L}\right)^{2(N+1)}
\end{aligned}
$$

For high reflective coating with a large number of periods $N$ (i.e. $N>10$ ), Eq. (4) reads

$\varphi \simeq \arctan \left(\frac{2 \eta_{\text {air }} / \eta_{L} \cos \delta_{L}^{\prime} \sin \delta_{L}^{\prime}}{\eta_{\text {air }}^{2} / \eta_{L}^{2} \sin ^{2} \delta_{L}^{\prime}-\cos ^{2} \delta_{L}^{\prime}}\right)$

In this case, the phase shift depends only on the tuned layer

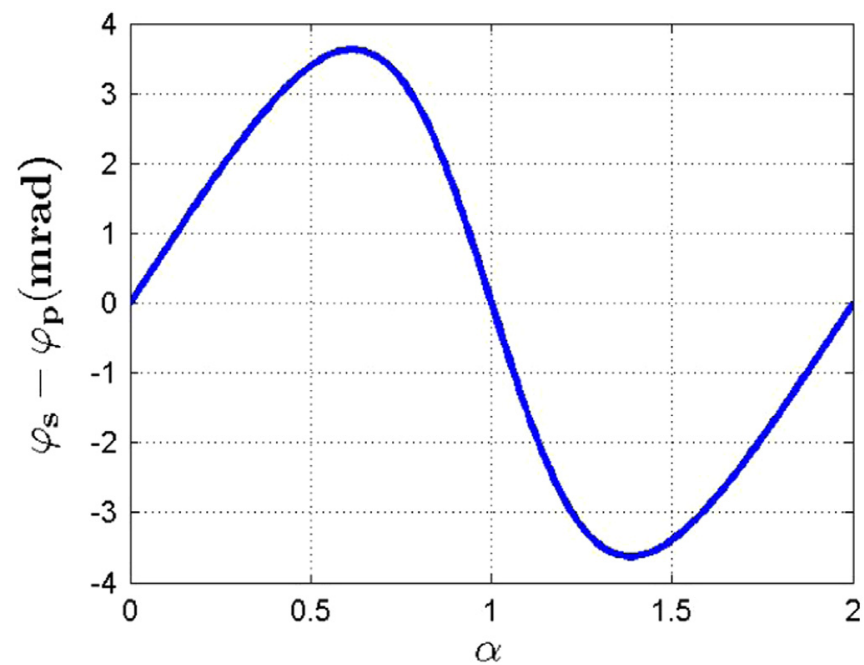

Fig. 1. Difference between the s- and p-wave reflection phase shifts $\left(\varphi_{s}-\varphi_{p}\right)$ as a function of the tuning coefficient $\alpha$ of the first layer.

phase factor $\delta_{L}^{\prime}$ and optical admittance $\eta_{L}$. Unlike the former, the latter depends on the polarization plane, thus inducing different phase shifts on reflection for both polarizations. The phase shift difference is given as a function of the tuning parameter $\alpha$ in Fig. 1. Phase shift differences up to $\pm 3.5 \mathrm{mrad}$ can be achieved with $\alpha=0.62$ or 1.38 . The effect of the birefringence was simulated using the model of Ref. [20] and found that it did not change significantly the curve of Fig. 1. Influence of thermal increase of the coatings on the phase shift difference was simulated and found to be of the order of $7.5 \times 10^{-6} \mathrm{rad} / \mathrm{K}$. This effect can thus be neglected.

This technique has been used to design the coating of the fourmirror planar bow-tie cavity in Section 3 .

\section{Stabilization method}

This section concentrates on four-mirror planar cavities, but the method can be extended for other topologies of cavities. The complex reflection coefficient for s- and p-wave in the basis attached to the incidence plane of mirror $i$ reads [17] $r_{i s, p}=\rho_{i s, p} \exp \left(i \varphi_{i s, p}\right)$ where $\rho_{i s, p}$ and $\varphi_{i s, p}(i \in[1,4])$ are respectively the amplitude and phase shift of the reflection coefficient for sand p-waves of the $i$ th mirror. The Jones vector circulating in the cavity is [17]

$\mathbf{V}_{\text {circ }}=\left(\begin{array}{cc}\frac{t_{1 s}}{1-\xi_{s} \exp \left(i \Psi_{s}\right)} & 0 \\ 0 & \frac{t_{1 p}}{1-\xi_{p} \exp \left(i \Psi_{p}\right)}\end{array}\right) \mathbf{V}_{0}$

where $t_{1 s, p}$ are the transmission factors of the input mirror for both s- and p-wave; $\xi_{s, p}=\rho_{1 s, p} \rho_{2 s, p} \rho_{3 s, p} \rho_{4 s, p}$, and $\Psi_{s, p}=$ $\varphi_{1 s, p}+\varphi_{2 s, p}+\varphi_{3 s, p}+\varphi_{4 s, p}+\varphi_{\text {space }}$, with $\varphi_{\text {space }}$ being the spatial propagation phase shift, $\mathbf{V}_{0}^{\mathrm{T}}=\left(V_{0 s}, V_{0 p}\right)$ normalized to 1 is the incident Jones vector of polarization. The intensity circulating in the cavity is

$\frac{I_{c i r c}}{I_{i n}}=\left|\frac{t_{1 s}}{1-\xi_{s} \exp \left(i \Psi_{s}\right)} V_{0 s}\right|^{2}+\left|\frac{t_{1 p}}{1-\xi_{p} \exp \left(i \Psi_{p}\right)} V_{0 p}\right|^{2}$.

Ignoring the losses in the cavity, the complex amplitude of the reflected wave is $[14,21]$ 
Table 1

Parameters of cavity mirrors.

\begin{tabular}{lllll}
\hline Mirror & M1 & M2 & M3 & M4 \\
\hline Diffusion (ppm) & 2 & 2.5 & 2 & 3 \\
Absorption (ppm) & 1 & 0.68 & 0.62 & 0.64 \\
Transmission (ppm) & 1060 & 410 & 334 & 375 \\
Nominal $\varphi$ (rad) & $\pi-0.0018$ & $\pi-0.0028$ & $\pi-0.0028$ & $\pi-0.0028$ \\
\hline
\end{tabular}

$E_{s, p}^{\mathrm{r}}=E_{s, p}^{\mathrm{i}}\left[\sqrt{R_{1}}-\frac{T_{1}}{\sqrt{R_{1}}} \frac{R \exp \left(i \Psi_{s, p}\right)}{1-R \exp \left(i \Psi_{s, p}\right)}\right] \equiv E_{s, p}^{\mathrm{i}} F\left(\Psi_{s, p}\right)$

where $R_{1}$ and $T_{1}$ are the reflectivity and transmission of the cavity input mirror respectively, $R=\sqrt{R_{1} R_{2} R_{3} R_{4}}$ is the cavity effective reflection coefficient, and $\Psi$ is the total phase shift after one cavity round-trip different for $\mathrm{s}$ - and $\mathrm{p}$-wave.

The proposed procedure is demonstrated on a four-mirror planar bow-tie cavity made of two planar mirrors (M1 and M2) and two spherical mirrors (M3 and M4) shown in Fig. 3. M1 also acts as the coupling mirror. The mirror coating design has been done according to Section 2. For each mirror the tuning coefficient $\alpha$ has been adjusted to provide an optimal coupling and a finesse 3000. As a result, a round-trip phase shift is induced.

Parameters of the mirrors are presented in Table 1 . Nominal $\varphi$ is the design phase shift in reflection between s- and p-wave. The total phase shift difference $\Psi_{s}-\Psi_{p}$ for s- and p-wave approximates 10 mrad. The cavity is $1.68 \mathrm{~m}$ long. The circulating intensity as a function of $\Psi_{s}$ is shown in Fig. 2 for various values of $\Psi_{s}-\Psi_{p}$. Two polarization peaks are observed when the phase difference is larger than 2 mrad.

In our design (see Fig. 3) a quarter wave plate and a polarizing beam splitter (PBS) are placed in the reflection path of the input

a

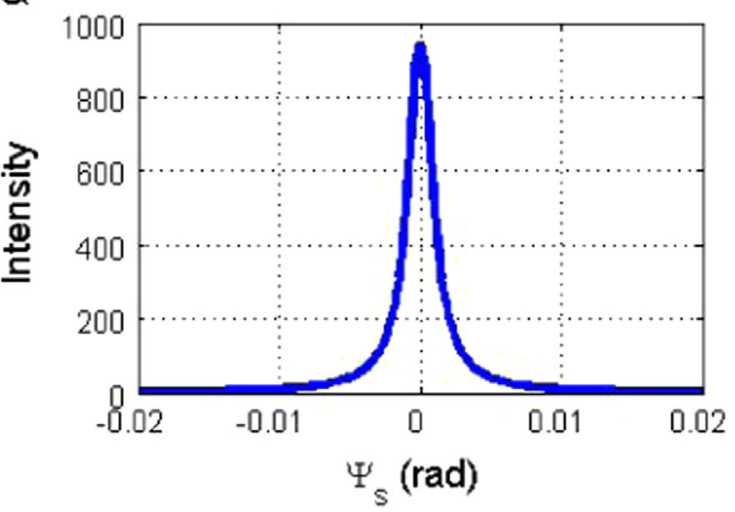

C

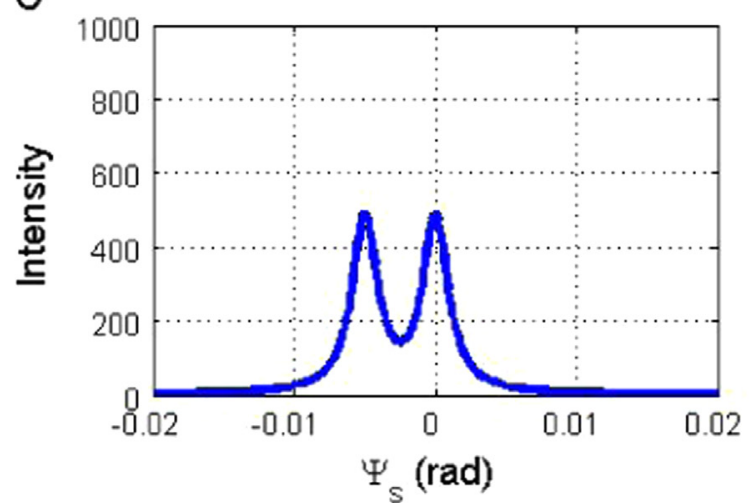

mirror. The quarter wave plate is rotated so that the angle between the fast axis of assembly and the optical table is $45^{\circ}$. The difference of the two polarization intensities obtained from reflection and transmission of the PBS acts as an error signal used for the feedback system.

A linear polarization rotated at $45^{\circ}$ is used as injection such that the reflection field reads

$\mathbf{E}^{\mathrm{r}} \propto\left(\begin{array}{c}F\left(\Psi_{p}\right)+e^{-i \frac{\pi}{2}} F\left(\Psi_{s}\right) \\ e^{-i \frac{\pi}{2} F\left(\Psi_{p}\right)+F\left(\Psi_{s}\right)}\end{array}\right) \equiv\left(\begin{array}{c}E_{p}^{\mathrm{r}} \\ E_{s}^{\mathrm{r}}\end{array}\right)$

where $F$ is the reflection amplitude of the optical cavity defined in Eq. (10). The signal $\left|E_{s}^{\mathrm{r}}\right|^{2}-\left|E_{p}^{\mathrm{r}}\right|^{2}$ crosses zero at the maximum of the resonance peaks as shown in Fig. 4. By choosing the sign of the slope of the error signal, one can select different polarization peaks. The slope of the error signal,

$K=\left.\frac{\partial\left(\left|E_{s}^{\mathrm{r}}\right|^{2}-\left|E_{p}^{\mathrm{r}}\right|^{2}\right)}{\partial \Psi_{S}}\right|_{\Psi_{s}=0}$

is maximized when $\Psi_{S}-\Psi_{p}=\pi$. However, the value of the slope is equal to $99 \%$ of its maximum as soon as $\left|\Psi_{s}-\Psi_{p}\right|>10 \mathrm{mrad}$. We estimate the variation of the phase shift due to the thermal effect in a zero-order QWP accounting for the change of the refractive indices and thickness with temperature to be of $3 \times 10^{-3} \mathrm{rad} / \mathrm{K}$. Thus only in the presence of very large ( $100 \mathrm{~K})$ temperature increase of the QWP, which is judged to be unlikely, a sensible effect on the error signal would be noticeable. However, this error signal was simulated and found to be not much distorted so that it can still be used to lock the cavity.

b

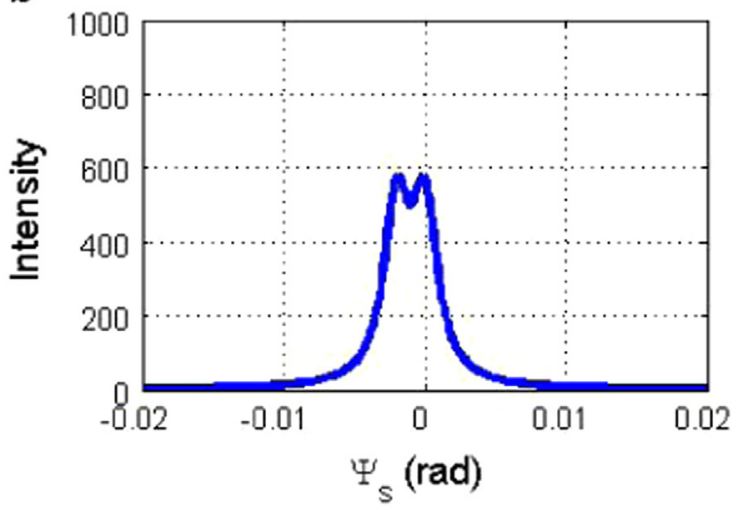

d

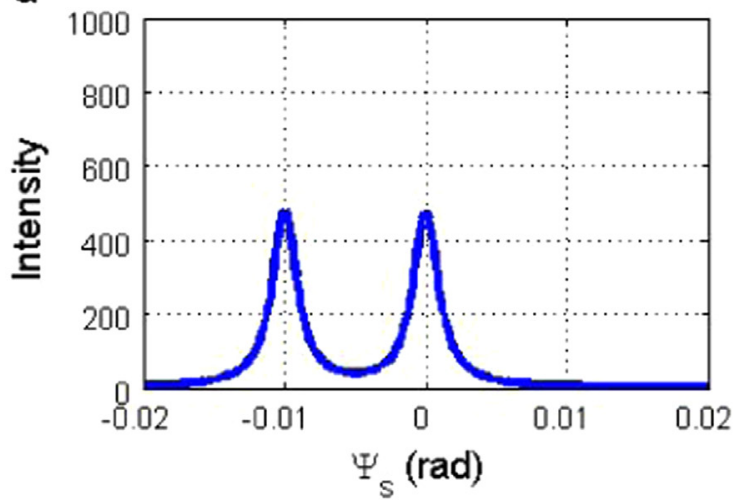

Fig. 2. Cavity circulating intensity as a function of $\Psi_{s}$. The four plots correspond respectively to $\Psi_{s}-\Psi_{p}=($ a) $0 \mathrm{mrad}$, (b) $2 \mathrm{mrad}$, (c) $5 \mathrm{mrad}$, and (d) $10 \mathrm{mrad}$. 


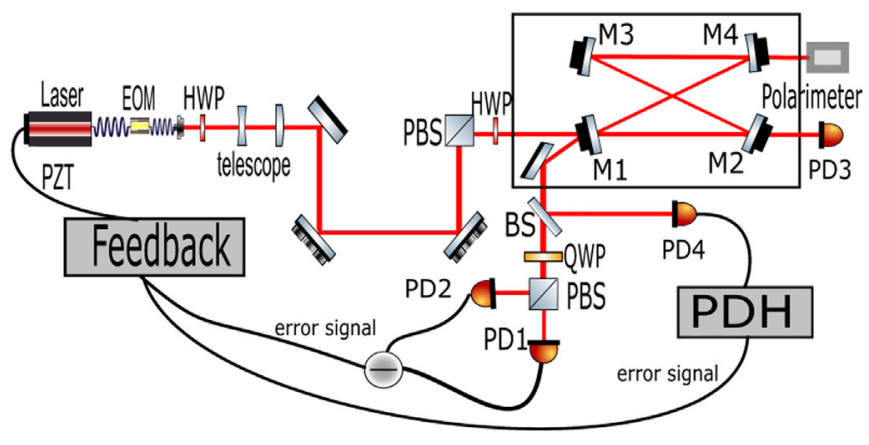

Fig. 3. Experiment setup. M1, M2, M3 and M4 are the Fabry-Perot cavity mirrors. PD1, PD2, PD3 and PD4 are photodiodes. EOM is an electro-optic modulator. HWP is a half-wave plate. QWP is a quarter wave-plate. PBS is a polarizing beam splitter. BS is a beam splitter.

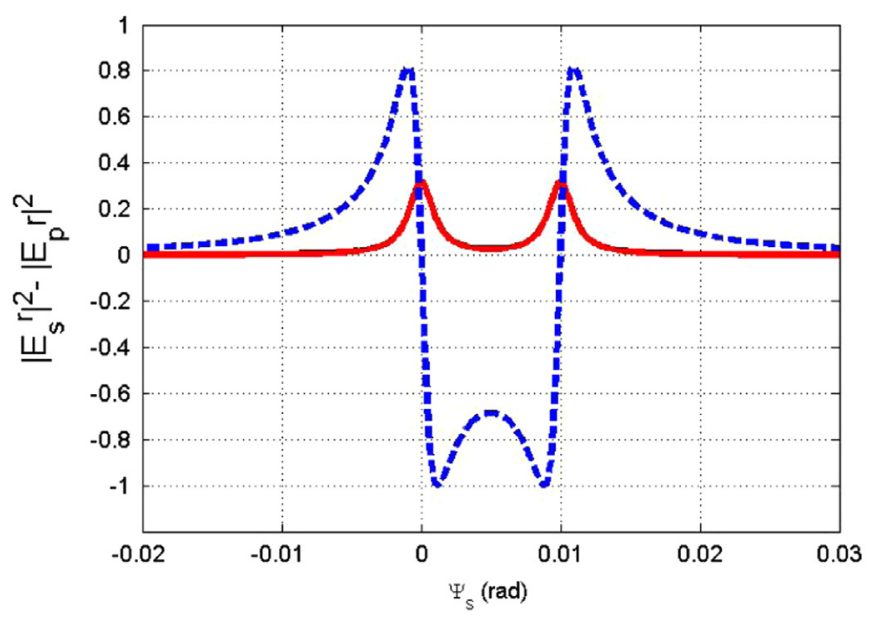

Fig. 4. Intensities of the cavity resonance peaks (solid line) and error signal (dashed line).

\section{Experiment}

An experiment was made to test this method as shown in Fig. 3. The pulsed laser used in the experiment is the Menlo Orange $\mathrm{Yb}$ Laser with $178.5 \mathrm{MHz}$ repetition rate and about $8 \mathrm{~nm}$ linewidth at $1030 \mathrm{~nm}$ central wavelength. A half-wave plate is placed upstream the injection in the cavity to produce a linearly polarized laser beam and balance the s- and p-wave intensities. The transmission intensity is monitored by the photodiode PD3. The cavity reflected field is directed onto a beamsplitter. Half of the intensity is measured by the photodiode PD4 used in the PDH technique. The other half of the intensity goes through a quarter wave-plate rotated at $45^{\circ}$ and a PBS separates the two polarization states. The intensities of these two beams are measured by the photodiodes PD1 and PD2. Their output is amplified in a differential amplifier which produces the error signal for the feedback.

The experimental error signal is shown in Fig. 5, in good qualitative agreement with calculations of Section 3. Two separated peaks are seen on the transmitted signal during laser frequency scan. The noise of the signals just comes from the pulsed laser phase noise alone. This error signal was used to successfully lock the laser on the cavity as shown in Fig. 6 . The bottom black curve is the voltage applied to the piezo electric transducer (PZT) of the laser cavity to adjust the frequency. In this case, the residual noise is effectively due to limited bandwidth of the PZT actuator. A good stability was observed for several hours. During the lock, the polarization of the light in transmission of M4 is measured with a

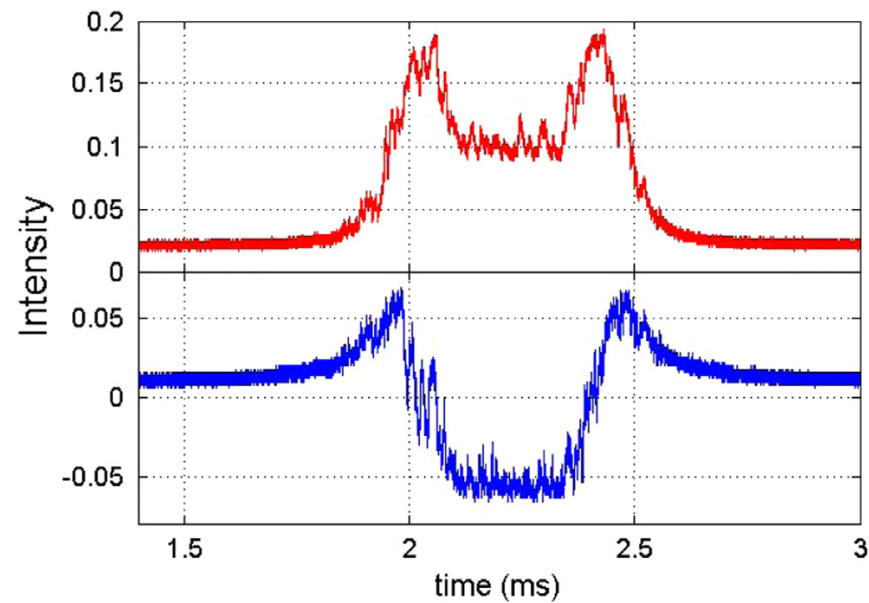

Fig. 5. When PZT of the laser is adjusting the frequency, the intensity of transmission (top red curve) and error signal (bottom blue curve) are obtained. (For interpretation of the references to color in this figure caption, the reader is referred to the web version of this paper.)

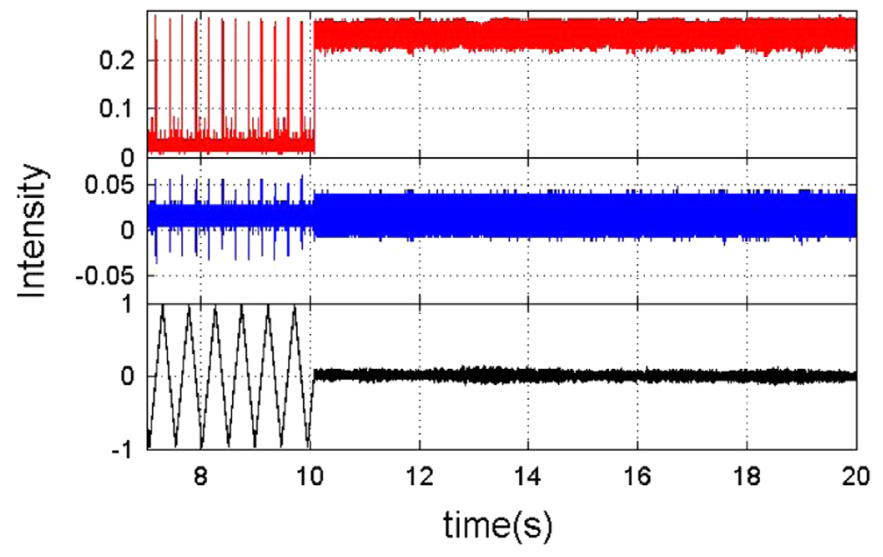

Fig. 6. Transmission (top red curve), error (middle blue curve), and PZT signals (bottom black curve) evolution over $20 \mathrm{~s}$. Lock starts at $10 \mathrm{~s}$. (For interpretation of the references to color in this figure caption, the reader is referred to the web version of this paper.)

polarimeter. In this case, alternance between horizontal and vertical polarizations is observed after a simple sign inversion of the feedback gain of the loop. In both cases the degree of linear polarization is larger than $99.5 \%$.

Different s- and p-wave intensities can be injected in the cavity by rotating the input half-wave plate. The possibility to have asymmetric amplitudes is crucial to reduce the power loss induced by the second polarization circulating in the cavity. The lock is still successful even though one peak is much smaller than the other one as shown in Fig. 7. During the experiment, it is necessary to balance the error signal to zero by rotating the quarter waveplate. The lock is very sensitive to the rotation.

This locking method is qualitatively compared to the well established PDH technique in Fig. 7. Polarization lock and PDH technique error signals are thus measured at the same time. The polarization lock method can be easily implemented without any frequency modulation. The two error signals both cross zero at the maximum of the resonance peaks and look similar, however, the PDH technique cannot discriminate different polarizations since both slopes of the error signals have the same signs. Besides, since our error signal is built on a similar way as in Ref. [16] where a quantitative comparison is provided, we expect that locking performance will be similar to $\mathrm{PDH}$. 


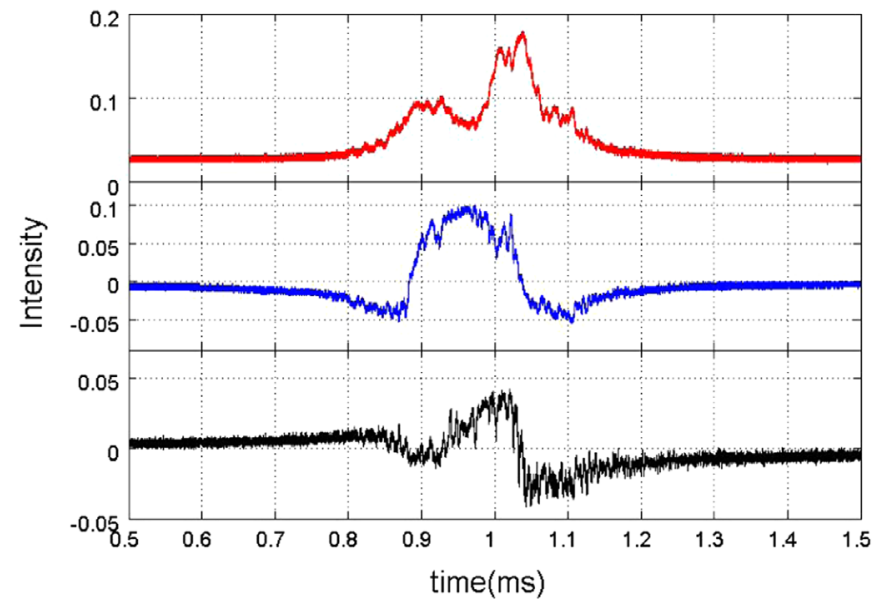

Fig. 7. Experimental polarization (middle blue curve) and PDH (bottom black curve) error signals obtained with non-symmetric resonant peaks (top red curve). (For interpretation of the references to color in this figure caption, the reader is referred to the web version of this paper.)

\section{Conclusion}

A new method to lock a laser on an optical cavity is proposed. Special design of coating layers of the cavity mirrors and laser non-nominal incidence angle lead to enough s- and p-wave phase detuning to generate two resonance peaks in the cavity. This polarization property can be used to generate an error signal and lock the laser to the cavity. Theoretical calculation and experiment results proved its feasibility.

\section{References}

[1] J. Rifkin, R.J. Loewen, R.D. Ruth, Apparatus, System, and Method for High Flux, Compact Compton X-ray Source, US Patent 7,277,526, October 2, 2007.

[2] Z. Huang, R.D. Ruth, Laser-electron storage ring, Phys. Rev. Lett. 80 (5) (1998) 976.

[3] J. Urakawa, K. Kubo, N. Terunuma, T. Taniguchi, Y. Yamazaki, K. Hirano, M. Nomura, I. Sakai, M. Takano, N. Sasao, et al., Electron beam cooling by laser,
Nucl. Instrum. Methods Phys. Res. Section A: Accel. Spectrom. Detect. Assoc. Equip. 532 (1) (2004) 388-393.

[4] M.J. Thorpe, J. Ye, Cavity-enhanced direct frequency comb spectroscopy, Appl. Phys. B 91 (3-4) (2008) 397-414.

[5] K.D. Moll, R.J. Jones, J. Ye, Output coupling methods for cavity-based highharmonic generation, Opt. Express 14 (18) (2006) 8189-8197.

[6] G. Moortgat-Pick, T. Abe, G. Alexander, B. Ananthanarayan, A. Babich, V. Bharadwaj, D. Barber, A. Bartl, A. Brachmann, S. Chen, et al., Polarized positrons and electrons at the linear collider, Phys. Rep. 460 (4) (2008) 131-243.

[7] I. Pupeza, S. Holzberger, T. Eidam, H. Carstens, D. Esser, J. Weitenberg, P. Rußbüldt, J. Rauschenberger, J. Limpert, T. Udem, et al., Compact high-repetition-rate source of coherent 100 ev radiation, Nat. Photon. 7 (8) (2013) 608-612.

[8] H. Sakai, Y. Honda, N. Sasao, S. Araki, Y. Higashi, T. Okugi, T. Taniguchi, J. Urakawa, M. Takano, Measurement of an electron beam size with a laser wire beam profile monitor, Phys. Rev. Spl. Top. Accel. Beams 4 (2) (2001) 022801.

[9] I. Agapov, G. Blair, M. Woodley, Beam emittance measurement with laser wire scanners in the international linear collider beam delivery system, Phys. Rev. Spl. Top. Accel. Beams 10 (11) (2007) 112801.

[10] D. Shaddock, M. Gray, D. McClelland, Frequency locking a laser to an optical cavity by use of spatial mode interference, Opt. Lett. 24 (21) (1999) 1499-1501.

[11] R. Drever, J.L. Hall, F. Kowalski, J. Hough, G. Ford, A. Munley, H. Ward, Laser phase and frequency stabilization using an optical resonator, Appl. Phys. B 31 (2) (1983) 97-105.

[12] E.D. Black, An introduction to Pound-Drever-Hall laser frequency stabilization, Am. J. Phys. 69 (1) (2001) 79-87.

[13] B. Slagmolen, D. Shaddock, M. Gray, D. McClelland, Frequency stability of spatial mode interference (tilt) locking, IEEE J. Quantum Electron. 38 (11) (2002) 1521-1528.

[14] T. Hansch, B. Couillaud, Laser frequency stabilization by polarization spectroscopy of a reflecting reference cavity, Opt. Commun. 35 (3) (1980) 441-444.

[15] Y. Honda, H. Shimizu, M. Fukuda, T. Omori, J. Urakawa, K. Sakaue, H. Sakai, N. Sasao, Stabilization of a non-planar optical cavity using its polarization property, Opt. Commun. 282 (15) (2009) 3108-3112.

[16] P. Asenbaum, M. Arndt, Cavity stabilization using the weak intrinsic birefringence of dielectric mirrors, Opt. Lett. 36 (19) (2011) 3720-3722.

[17] F. Zomer, Y. Fedala, N. Pavloff, V. Soskov, A. Variola, Polarization induced instabilities in external four-mirror Fabry-Perot cavities, Appl. Opt. 48 (35) (2009) 6651-6661.

[18] F. Brandi, F. Della Valle, A. De Riva, P. Micossi, F. Perrone, C. Rizzo, G. Ruoso, G. Zavattini, Measurement of the phase anisotropy of very high reflectivity interferential mirrors, Appl. Phys. B: Lasers Opt. 65 (3) (1997) 351-355.

[19] H.A. Macleod, Thin-Film Optical Filters, CRC Press, Tucson, Arizona, USA, 2010 (Chapter 2).

[20] F. Bielsa, A. Dupays, M. Fouché, R. Battesti, C. Robilliard, C. Rizzo, Birefringence of interferential mirrors at normal incidence, Appl. Phys. B 97 (2) (2009) 457-463.

[21] M. Born, E. Wolf, Principles of Optics: Electromagnetic Theory of Propagation, Interference and Diffraction of Light, Cambridge University Press, New York, USA, 1999. 